\title{
Privatisation et logique marchande dans l'éducation aux États-Unis
}

Privatization and marketization in US education

Privatización y lógica del mercado en la educación en Estados Unidos

Christopher Lubienski, T. Jameson Brewer et Jin Ah Kim

Traducteur : Hélène Bréant

\section{(2) OpenEdition}

Journals

Édition électronique

URL : https://journals.openedition.org/ries/9110

DOI : 10.4000/ries. 9110

ISSN : 2261-4265

Éditeur

France Education international

Édition imprimée

Date de publication : 1 décembre 2019

Pagination : 57-66

ISBN : 9782854206258

ISSN : 1254-4590

Référence électronique

Christopher Lubienski, T. Jameson Brewer et Jin Ah Kim, «Privatisation et logique marchande dans l'éducation aux États-Unis ", Revue internationale d'éducation de Sèvres [En ligne], 82 | décembre 2019, mis en ligne le 01 décembre 2021, consulté le 14 janvier 2022. URL : http://journals.openedition.org/ ries/9110; DOI : https://doi.org/10.4000/ries.9110 


\title{
Privatisation et logique marchande dans l'éducation aux États-Unis*
}

\section{Christopher Lubienski \\ Indiana University}

\section{T. Jameson Brewer}

University of North Georgia

\author{
Jin Ah Kim \\ Indiana University
}

Si les chercheurs ont décrit les tendances mondiales qui vont dans le sens de la privatisation de l'éducation, ils ont aussi observé des modèles spécifiques et des voies singulières qui diffèrent suivant les contextes (Verger, Fontdevila et Zancajo, 2016). Le cadre de Ball et Youdell (2007) sur les formes endogènes et exogènes de privatisation est particulièrement pertinent pour comprendre la multiplicité des voies qu'empruntent les intérêts privés qui s'imposent de plus en plus face aux systèmes étatiques. Il montre ce faisant comment la promotion de la privatisation est doublement assurée non seulement à travers l'élaboration des politiques publiques mais aussi par l'émulation des modes, pratiques et valeurs du secteur privé. Quoique les États-Unis soient, en substance, le reflet de ces tendances mondiales, en nous appuyant sur la notion de pénétration de la logique marchande pour mieux comprendre les contextes, les dynamiques et les « biens », nous mettrons en lumière trois facteurs qui sont particulièrement prononcés - sinon uniques - aux États-Unis : la privatisation de la décision en matière de politiques éducatives, le développement de marchés qui concernent tous les types d'établissements et la privatisation du but même de l'éducation qui en découle.

Dans la prochaine section, nous esquisserons les lignes de faille traditionnelles entre secteur public et secteur privé qui traversent l'enseignement élémentaire et secondaire aux États-Unis, et nous examinerons les défis que pose depuis peu à la gouvernance publique l'éducation financée par de l'argent public. Ce faisant, nous mettons en évidence certaines des formes les plus communes de pénétration du privé dans le secteur public. Nous décrirons ensuite les évolutions intervenues en matière d'élaboration des politiques publiques et d'enseignement dans le contexte américain, en montrant aussi comment la privatisation intervient dans l'élaboration des politiques publiques et dans la marchandisation de l'éducation. Cependant, mettre l'accent sur le contexte marchand dans lequel évolue l'école elle-même explique bien mieux les forces auxquelles sont confrontés différents types d'établissements. Nous soulignerons brièvement les conséquences politiques, institutionnelles, financières, sociales et académiques de ces tendances avant d'aborder, en conclusion, les facettes de l'éducation entendue comme un bien public au sein d'un modèle devenu marchand.

* Article traduit par Hélène Bréant. 


\section{DÉPLACEMENT DES LIMITES ENTRE LES SECTEURS ÉDUCATIFS PUBLIC ET PRIVÉ}

Du fait essentiellement de la séparation constitutionnelle de l'Église et de l'État aux États-Unis, le financement public des écoles privées - gérées en grande majorité par des organisations confessionnelles - fait traditionnellement l'objet d'une interdiction relativement stricte. Ce facteur a, dans une large mesure, préempté des changements majeurs vers la privatisation. Au XIX siècle, de nombreux États sont allés au-delà de la Constitution fédérale et ont strictement interdit l'allocation de fonds publics à des fins religieuses. Néanmoins, en 2002, la Cour suprême américaine a estimé que les chèques éducation ( « vouchers ») n'enfreignaient pas la Constitution fédérale puisque l'argent public est employé par des individus qui font des choix privés et affectent cet argent à des établissements privés. Bien que certains États conservent des dispositions moins libérales dans leurs Constitutions respectives, nombre de politiques publiques ont récemment effacé le fossé traditionnel entre les secteurs éducatifs public et privé, ce qui donne lieu à des transferts d'argent des établissements publics vers notamment :

- les «charter schools": financées par le secteur public mais gérées par le privé, ces écoles indépendantes sont techniquement publiques, quoiqu'un grand nombre d'entre elles soient gérées par des opérateurs à but lucratif. Presque toutes réduisent le contrôle démocratique direct de l'enseignement financé par la puissance publique, au profit d'une gestion assumée par des entités non élues et qui va de pair avec une responsabilisation des parents, considérés comme autant de consommateurs, empruntée au secteur marchand. Beaucoup présentent un comportement organisationnel propre aux organisations privées.

- les chèques éducation : ces chèques financés par de l'argent public et couvrant tout ou partie des frais de scolarité dans les écoles privées prolifèrent depuis le début des années 1990 .

- les programmes similaires au chèque éducation : comme de nombreux États maintiennent l'interdiction de financer les chèques éducation sur les deniers publics, les décideurs politiques ont cherché d'autres possibilités, telles que des comptes d'épargne individuels dédiés à l'éducation et des crédits d'impôt appliqués aux frais de scolarité qui contournent cette interdiction, mais qui, du point de vue fonctionnel, équivalent aux chèques éducation.

Voici d'autres exemples courants de la pénétration des intérêts privés dans l'enseignement aux États-Unis :

- consultance et formation : les organisations du champ éducatif étant de plus en plus encouragées à ressembler à des entreprises, les acteurs du secteur privé offrent une assistance en accompagnement ou en gestion des changements organisationnels dans des domaines comme le développement du leadership, la gestion des ressources humaines et la sous-traitance. D'autres organisations privées prennent un caractère de plus en plus prévalent en matière de préparation à l'enseignement et de développement professionnel des enseignants.

- gestion de l'éducation : ces organisations à but lucratif et non lucratif exploitent des écoles et des systèmes scolaires - des chaînes de charter schools, par 
exemple - en assurant leur administration, l'enseignement, le curriculum, la gestion des inscriptions et d'autres fonctions traditionnellement assumées par les circonscriptions scolaires publiques. Elles sont monnaie courante dans le secteur scolaire en ligne, et nombre d'entre elles ont été amenées pour « redresser » des écoles publiques aux piètres performances.

- prestataires de services complémentaires : des entités à but lucratif se proposent de décharger les écoles publiques de fonctions accessoires allant de la gestion du personnel ou de l'assistance au curriculum à l'aide au transport, à la restauration scolaire et aux modes de garde des enfants.

Un ensemble relativement restreint d'objectifs de politiques publiques est avancé pour justifier ce type de politiques, le plus fréquent étant l'amélioration de l'efficacité et de l'efficience des établissements scolaires. Mais d'aucuns postulent aussi que la privatisation œuvre à une plus grande équité, puisque les enfants ne sont plus assignés à des écoles publiques médiocres. Cependant, d'autres zélateurs de la privatisation recherchent tout simplement une plus grande liberté de choix des familles ou la dérégulation du contrôle gouvernemental comme des fins en soi. Toutefois, quels qu'en soient les ressorts, les politiques susmentionnées reposent sur des critères souvent utilisés par les chercheurs pour comprendre la privatisation à l'œuvre dans le système scolaire américain, plaçant principalement l'accent sur l'enjeu de la propriété des écoles ou des services. Néanmoins, ces points de vue omettent de prendre en compte des facteurs moins tangibles à l'origine d'une forme de contrôle privé plus profonde que celle qui consiste simplement à transférer un nombre d'enfants relativement faible d'un établissement public à une école indépendante ou privée.

\section{Privatisation et logiQue MARCHANDE DANS L'ÉDUCATION}

Si d'aucuns considèrent que la privatisation à l'œuvre dans le système scolaire américain est due au départ d'élèves qui quittent les écoles publiques gérées par les districts, ces phénomènes ne constituent pas en réalité une forme de privatisation des écoles. Comme l'explique Diane Ravitch, critique de ces réformes (1996) :

[ces politiques] ne sont pas autant de pas vers la privatisation, comme l'affirment leurs détracteurs. Ces établissements scolaires sont financés par de l'argent public, autorisés par la loi, et ils rendent des comptes à la puissance publique. La privatisation se produit au contraire lorsqu'une entité publique est transférée aux mains de propriétaires privés qui rendent des comptes à leurs actionnaires, pas à la puissance publique. À l'évidence, ce n'est le cas ni des charter schools ni des écoles gérées par des opérateurs privés.

Cependant, mettre l'accent de cette manière sur les écoles elles-mêmes - leur régime de propriété, leur financement et leur gouvernance - présente des limites et ne contribue guère à mettre en lumière la progression substantielle de l'entreprise de remodelage de l'éducation par des intérêts privés. En fait, la privatisation se produit à un niveau beaucoup plus profond que celui du simple transfert de la propriété ou du financement des établissements scolaires, souvent dans des espaces qui vont au-delà des établissements eux-mêmes. 


\section{L'élaboration des politiques publiques est privatisée}

L'élaboration des politiques publiques telles qu'on l'entend habituellement (et de façon simpliste) implique que des décideurs élus délibèrent en vue d'instaurer des règles et des règlements qui reflètent les préférences d'un public plus large. Bien évidemment, les considérations politiques, idéologiques, et celles qui ont trait à des intérêts particuliers ternissent l'idéal de la construction de politiques publiques comme processus rationnel. De nombreuses voix ont ainsi affirmé que les syndicats d'enseignants avaient dominé les politiques publiques éducatives aux États-Unis, réorientant les bénéfices de l'enseignement public vers leurs adhérents et non vers les enfants ou, plus largement, la société. Dans le même temps, des fondations philanthropiques privées telles que Carnegie, Ford et Annenberg ont investi des sommes colossales pour améliorer les écoles publiques dans le sens de leurs propres agendas. Toutefois, ces dernières années, des intérêts privés - parfaitement illustrés par ces tendances dans le champ philanthropique - ont modifié les stratégies en cherchant à constituer une palette alternative d'institutions qui supplantent l'éducation publique au lieu de la soutenir. Ils ont réussi à le faire en s'immisçant dans le processus d'élaboration des politiques publiques éducatives, qu'ils ont pris en otage (Layton, 2014 ; Reckhow, 2013).

La privatisation de la décision en matière de politiques publiques se produit de multiples manières. Assurément, des organisations privées font du lobbying et 60 contrôlent l'agenda des politiques publiques, empruntant des canaux d'influence plus classiques. Mais l'on assiste aussi à la sous-traitance de la législation à des organisations privées, non gouvernementales, telles que l'American Legislative Exchange Council (ALEC), et même au placement d'individus salariés par des sociétés privées à des postes publics. De manière générale, nous assistons à l'essor de réseaux d'influence sur ces politiques, souvent financés par des fondations philanthropiques, qui travaillent à faire converger les résultats de la recherche scientifique, la promotion, l'attention des médias et le soutien politique, et ce afin de faire progresser leurs agendas pour l'éducation, fondés sur le marché. L'ALEC offre par exemple au législateur public des modèles de textes prêts à l'emploi dans des domaines tels que les chèques éducation et les charter schools, externalisant et privatisant ainsi l'élaboration des politiques publiques dans leur essence même. De surcroît, on voit proliférer des think tanks - largement financés par les mêmes donateurs privés - qui créent, recueillent, mettent en forme et diffusent la recherche qui vient étayer les positions de leurs fondateurs en matière de politiques publiques (Lubienski, Brewer et LaLonde, 2016).

De la même manière, des organisations qui ont réussi à faire pénétrer le privé dans le métier d'enseignant, à l'instar de Teach For America (TFA), ont étendu les objectifs des organisations cherchant à influer sur le processus d'élaboration des politiques publiques. Par exemple, l'organisation politique Leadership for Educational Equity (LEE), émanation de TFA, se concentre spécifiquement sur le soutien aux ambitions politiques des anciens élèves de TFA. Elle leur fournit en effet une surveillance stratégique des campagnes politiques, un financement et la mise en réseau des candidats. TFA constitue donc pour ses anciens élèves une plate- 
forme bien dotée financièrement et bien connectée, qui leur permet d'accéder à de nombreuses responsabilités publiques, y compris dans les conseils d'administration des établissements scolaires, les parlements des États, aux postes de gouverneurs et au Congrès. De même, TFA promeut « The Capitol Hills Fellows initiative », financée par un capital-risqueur de la Silicon Valley, afin de permettre aux anciens élèves de TFA de travailler pour le Congrès en tant qu'agents rémunérés.

\section{Le système scolaire est transformé en marché}

Si ces phénomènes à l'œuvre dans la privatisation de l'élaboration de politiques publiques ne manquent pas d'intérêt, ils ne nous apprennent pas grand-chose sur le type de politiques visées par ces nouvelles façons de procéder. Comme nous l'avons précisé plus haut, il serait difficile d'arguer que les auteurs des réformes en éducation qui utilisent un mode privatisé d'élaboration des politiques publiques ont pour fin la privatisation pure et simple des écoles - du moins au sens classique du terme. À l'évidence, certaines écoles publiques ont été confiées (au moins provisoirement) à des gestionnaires privés dont on attend qu'ils les " redressent » pour les rendre plus efficaces. Et un grand nombre de villes ont fermé leurs écoles publiques en sous-effectif, tout en encourageant la prolifération de charter schools gérées par le privé. À vrai dire, certains États comme l'Indiana ont même contraint les circonscriptions scolaires à vendre pour une bouchée de pain les bâtiments inutilisés à des opérateurs « charter ». Mais au lieu d'un mouvement général de transfert de propriété ou de contrôle des écoles publiques à des intérêts privés - comme celui auquel on aurait assisté, selon certains, avec la privatisation d'entreprises publiques partout dans le monde dans les années 1980, ce que nous observons est en fait une tendance bien plus vaste et, potentiellement, d'une portée bien plus importante : l'établissement d'un marché de l'éducation. Ce concept explicite les changements qui surviennent vers des environnements institutionnels de type marchand, lesquels soumettent les écoles, indépendamment de leur type ou de leur orientation première, à des pressions concurrentielles qui les incitent à agir davantage comme des entreprises privées. Ces forces motrices de la " marchéisation » sont le fruit d'agendas de politiques publiques promus par des auteurs de réformes éducatives par le truchement des méthodes d'élaboration de politiques publiques privatisées que nous avons décrites. Ces politiques comprennent des mesures telles que la liberté de choix de l'établissement, la dérégulation des écoles (avec, par exemple, une plus grande autonomie des charter schools) et le financement transférable (avec les chèques éducation). Si les efforts de leurs thuriféraires diffèrent autour de certaines politiques en particulier, ces politiques publiques dans leur ensemble confèrent généralement aux écoles un statut d'entités de type marchand et attribuent aux parents un rôle de consommateurs sur le marché scolaire. Du côté de la demande, les familles sont encouragées à sélectionner la meilleure école pour leur enfant (sauf à courir le risque qu'il soit à la traîne). Du côté de l'offre, les établissements scolaires disposent d'une autonomie plus grande pour innover, agir de manière plus entrepreneuriale et s'engager dans une logique concurrentielle afin d'attirer les élèves et le financement per capita que ces derniers leur apportent. 
À cet égard, l'école publique, par exemple, ouverte à tous les élèves quelles que soient leurs ressources, est confrontée au risque de perdre des élèves (et des subventions) au profit des charter schools et des écoles privées; elle doit donc s'engager dans cette compétition pour attirer et retenir les élèves. Indépendamment du statut de l'école, qu'elle soit publique, privée ou privatisée, l'environnement institutionnel devenu marchand incite les écoles à adopter un comportement organisationnel tourné vers des stratégies empruntées aux entreprises du secteur privé. Nous voyons donc des écoles publiques faire de la publicité pour leurs services, par exemple, quoiqu'elles ne s'adressent là qu'aux familles les plus « désirables ». Nous observons des charter schools, fondées sur les principes de l'engagement auprès de la collectivité ou des bienfaits pour la société, se tourner vers des approches pédagogiques ou disciplinaires qui excluent les élèves dont l'instruction coûte plus cher. Des écoles privées qui avaient été fondées pour répondre aux besoins de groupes confessionnels spécifiques changent leur mission afin de remplir les salles de classe et de maximiser les chèques éducation intégralement financés par les pouvoirs publics. En un mot, les environnements institutionnels transformés en marchés peuvent inciter tout type d'école, indépendamment de son régime de propriété, à agir davantage comme les entités privées à but lucratif.

\section{Le « bien » qu'est l'éducation est privatisé}

Si les auteurs des réformes en éducation ont recours à l'élaboration - privatisée - des politiques publiques pour faire avancer la transformation de l'école en marché plutôt que de privatiser directement les écoles publiques, le résultat final est la privatisation du but de l'enseignement public. L'éducation a été maintes fois décrite comme un "bien »- un bien public, privé, spécifique, commun, ou encore comme un bien " positionnel », par exemple. Tandis qu'on assiste à des débats intéressants sur le type de bien qu'est l'éducation, il est évident que l'enseignement public est habituellement traité par les décideurs politiques du monde entier comme un (quasi) bien public, ou du moins comme un bien doté de fortes externalités positives ou de bénéfices pour la société. C'est pourquoi les écoles publiques sont très accessibles, ouvertes à tous et (presque) gratuites, subventionnées et gérées par la puissance publique. En tant qu'institutions accessibles à tous, les écoles publiques - dans la majorité des cas - ne peuvent pas refuser des élèves de leur secteur, même si un élève pose de gros problèmes et qu'il coûte cher à l'établissement, et les élèves ne sont pas mis en concurrence pour pouvoir bénéficier de l'enseignement public. En retour, les écoles publiques sont censées apporter à la société des bénéfices plus étendus, notamment des citoyens mieux instruits, mieux informés, plus tolérants et plus unis, des niveaux plus élevés de développement culturel et économique, et des électeurs plus éclairés ou plus critiques. Si ces fins de l'éducation ont souvent été plus idéalisées que réellement atteintes dans les écoles publiques américaines, nul ne conteste le fait que le caractère de bien public de l'éducation s'est forgé dans les institutions de l'enseignement public.

Néanmoins, les écoles, aiguillonnées par l'élaboration privatisée de politiques publiques - souvent à l'instigation de réformateurs philanthropiques de l'éducation qui sont eux-mêmes des hommes d'affaires et qui semblent appréhender 
l'éducation en des termes issus de l'entreprise -, sont encouragées à agir davantage en entreprises et les parents incités à adopter une posture consumériste. Une telle redéfinition des rôles modifie les relations et les dynamiques de l'enseignement conçu comme un bien public accessible à tous. En tant qu'organisations qui cherchent à maximiser leurs profits en se faisant concurrence sur un marché, les écoles recourent souvent à des mesures incitatives afin d'attirer et de servir certains élèves, et en excluent d'autres qui pourraient nuire à leur position de marché. Effectivement, ces pratiques sont attestées ( $c f$. infra). Dans le même temps, les familles sont sommées de supporter les coûts de recherche (et souvent les coûts de transport), de soupeser les différentes options puis de sélectionner une école pour leur enfant, comme elles opteraient pour d'autres biens de consommation ou d'autres services. Il est notable que, sur un marché concurrentiel de consommateurs avec des prestataires plus ou moins désirables, certaines écoles sont particulièrement populaires, ce qui contraint dès lors les familles à se faire concurrence pour y accéder, que ce soit à travers les critères d'inscription, les processus d'admission, l'adéquation sur le plan académique, etc. En cela, l'éducation devient moins un bien commun comme la police ou les services d'incendie et de secours, et davantage un bien de consommation privatisé, à l'instar d'un vêtement soumis à une mode. Quoique les écoles aient toujours apporté aux apprenants des bénéfices individuels, souvent mis au regard des considérations pour la collectivité dans son ensemble, la privatisation du but même de l'éducation ne met en exergue que les aspects qui peuvent être consommés par des individus.

\section{LES EFFETS DE LA PRIVATISATION}

Comme nous l'avons constaté, la privatisation est la plus visible à la fois dans le processus d'élaboration des politiques éducatives et dans la transformation en marchandise de l'éducation entendue comme le bien préféré dans un modèle devenu marchand. Ces tendances ont plusieurs effets sur les dispositions institutionnelles (politiques et structurelles), financières et sociales autour de l'éducation, mais - cela pourra sembler surprenant - elles sont relativement tempérées, voire contreproductives, dans leurs impacts sur les résultats scolaires où, selon les réformateurs, elles devaient avoir les effets les plus nets.

La privatisation de la décision en matière de politiques éducatives a déjà eu des effets significatifs sur les institutions politiques. Les fondations philanthropiques privées sont devenues des acteurs politiques majeurs, non seulement en créant des canaux pour influer sur la décision classique en matière de politiques publiques, par exemple en finançant des candidats briguant un poste politique et des études promotionnelles (Au et Lubienski, 2016), mais aussi en inventant de nouveaux moyens d'élaborer directement des politiques, comme le fait la Fondation Bill \& Melinda Gates en apportant son soutien politique et financier à des organisations (souvent nouvelles) afin qu'elles puissent créer, promouvoir, encourager et même dicter de nouvelles politiques éducatives (Layton, 2014). Parmi les bénéficiaires les plus patents de ce phénomène figurent des organisations qui plaident pour des réformes fondées sur le marché, en particulier celles qui gèrent des charter schools, dont un grand nombre a atteint une échelle qui leur confère une influence politique substantielle. Effectivement, en bien des façons, ces organisations gestion- 
naires ont privatisé la gouvernance des écoles, dans la mesure où elles supplantent des districts scolaires pilotés de manière démocratique en dupliquant leurs fonctions à partir d'archétypes empruntés au monde de l'entreprise. Leurs écoles ne sont donc plus financées par les seuls contribuables, mais souvent aussi par des philanthropes privés, y compris des gestionnaires de fonds spéculatifs qui, quoique non élus, siègent ensuite dans des conseils d'administration qui procédaient jusqu'alors d'élections démocratiques.

Le but de l'éducation étant de plus en plus privatisé et repensé comme un bien individualisé, les politiques à l'origine de cet environnement marchand lient le financement public aux choix individuels des parents d'envoyer leurs enfants dans des établissements scolaires autres que des écoles publiques. Suivant de récentes décisions judiciaires et vu l'adhésion des décideurs publics à l'idée d'un financement transférable, il est possible que des montants astronomiques prélevés sur les deniers publics soient retirés des écoles publiques gérées démocratiquement. En outre, de nombreux programmes de charter schools et de chèques éducation profitent à des élèves dont les familles n'auraient de toute façon jamais envoyé leurs enfants dans une école publique, ce qui revient pour l'État à subventionner une décision prise par des familles aisées, et qu'elles auraient financée par leurs propres ressources, de scolariser leurs enfants dans des établissements privés. Dans l'ensemble, cela signifie que le financement public est de plus en plus privatisé, puisqu'il est effectivement accordé à des individus qui décident de l'usage qu'ils en feront pour leurs propres enfants, sans les niveaux élevés de responsabilité publique associés aux écoles publiques.

Si la concurrence générée dans ces contextes devenus marchands est censée encourager les écoles à être plus efficaces dans l'éducation des élèves, il n'est pas toujours évident que les écoles dédient leurs ressources à progresser dans leur mission fondamentale - l'éducation des enfants. En fait, au lieu de recruter des enseignants plus qualifiés ou d'investir dans le développement d'un curriculum plus efficace, de nombreux établissements scolaires - qu'ils soient publics, privés ou des charter schools - préfèrent investir dans des campagnes de marketing afin d'attirer des élèves (et le financement per capita y afférent).

De la même manière, l'un des effets troublants des charter schools et des chèques éducation pour les écoles privées est le rôle qu'ils jouent en accentuant la ségrégation raciale et la ségrégation de classe entre les élèves. Alors que l'impulsion qui présidait initialement au lancement des chèques éducation dans les années 1950 et 1960 avait été spécifiquement utilisée par les parents blancs comme un mécanisme de levier pour éviter l'intégration, la recherche a montré que des décennies de libre choix de l'école avaient entraîné, indépendamment des motifs qui y avaient présidé, une hyper ségrégation, soit du fait des modèles de prise de décision parentale, soit $\mathrm{du}$ fait des comportements organisationnels des écoles - comme le marketing, par exemple - qui sélectionnent ou excluent implicitement certaines catégories d'élèves (Vasquez Heilig, Brewer et Williams, 2019).

En plus d'exacerber la ségrégation, les conséquences de la privatisation et de l'adoption de la logique marchande en salle de classe sont à des années-lumière de ce qu'avaient escompté les auteurs des réformes en éducation. Tandis qu'ils étaient nombreux à attendre un accroissement d'innovation de la part des écoles soumises à la concurrence, chacun convient désormais que l'innovation se voit plus en matière 
de marketing, d'emploi et d'autres comportements organisationnels étrangers au cœur technique de l'enseignement et du curriculum (Lubienski, 2003). En outre, les résultats académiques de ces politiques ont été jusqu'à présent d'une pauvreté notoire, pour ne pas dire négatifs. La plupart des examens relatifs aux performances des charter schools montrent que leurs élèves se classent dans une fourchette proche de celle des écoles publiques dont la démographie est comparable. Mais presque toutes les recherches récentes sur les chèques éducation révèlent que les élèves qui sont envoyés vers un établissement privé notent des conséquences importantes et négatives sur leurs apprentissages par rapport à leurs pairs restés dans le système scolaire public (Lubienski et Malin, 2019).

\section{Perspectives}

Le bilan indigent de l'éducation transformée en marché scolaire aux ÉtatsUnis dans ses dimensions académiques, sociales et sur la base d'autres critères de mesure, dénote un certain nombre de problèmes substantiels liés aux prescriptions en matière de politiques avancées par l'élaboration (privatisée) de politiques publiques, tout du moins pour ce qui a trait aux promesses des réformateurs de faire progresser ces politiques en termes d'efficacité, d'équité, d'innovation, etc. Néanmoins, le changement profond dans le traitement réservé à l'éducation, à la fois par les décideurs politiques et par les parents, suggère un impact profond qui va au-delà des promesses d'une plus grande équité ou de meilleurs résultats. En fait, transformer le «bien » qu'est l'éducation peut véritablement incarner les buts ultimes des réformateurs, bien plus qu'une efficience accrue ou même que la gouvernance individualisée de l'enseignement public.

Pourtant, ces tendances que nous avons identifiées passent largement à côté de la question suivante : les caractéristiques de l'éducation entendue comme un bien public peuvent-elles être préservées à long terme dans un contexte transformé en marché scolaire? De facto, au cours des cinquante dernières années, théoriciens et réformateurs américains ont expliqué que l'enseignement public serait mieux géré par des voies autres que publiques, et ont affirmé que donner aux familles plus de choix consuméristes conduirait à un système scolaire plus efficace, innovant et équitable dans un cadre marchand. Toutefois, le bilan empirique suggère de manière croissante que l'enseignement public placé dans un contexte marchand n'offre aucun de ces avantages, sinon pour l'option consumériste considérée comme une fin en soi. Cela privatise au contraire le but même de l'éducation en privilégiant le souci parental de son propre enfant plutôt que la préoccupation légitime de la collectivité au sens large du terme - pour « les enfants des autres », comme le disait John Dewey en une maxime restée dans les annales.

\section{RÉFÉRENCES BIBLIOGRAPHIQUES}

AU W. et LUBIENSKI C. (2016). « The role of the Gates Foundation and the philanthropic sector in shaping the emerging education market: Lessons from the US on privatization of schools and education governance ». In A. Verger, C. Lubienski et G. Steiner-Khamsi (ed.), The global education industry. New York : Routledge, p. 27-43. 
BALL S. et YOUDELL D. (2007). Hidden privatisation in public education. Brussels : Education International.

LAYTON L. (2014, 7 juin). « How Bill Gates pulled off the swift Common Core revolution ». Washington Post. [En ligne : https://wapo.st/2szWfZW].

LUBIENSKI C. (2003). «Innovation in education markets: Theory and evidence on the impact of competition and choice in charter schools ». American Educational Research Journal, 40(2), p. 395-443.

LUBIENSKI C., BREWER T.J. et LALONDE P. (2016). "Orchestrating Policy Ideas: Philanthropies and Think Tanks in US Education Policy Advocacy Networks ». Australian Education Researcher, 43 (1), p. 55-73.

LUBIENSKI C. et MALIN J.R. (2019). "School vouchers expand despite evidence of negative effects ». The Conversation. [En ligne : https://bit.ly/2PpOWNx].

RAVITCH D. (1996, février). «In deep denial », The New Democrat, p.21-23.

RECKHOW S. (2013). Follow the money: How foundation dollars change public school politics. Oxford : Oxford University Press.

VASQUEZ HEILIG J., BREWER T. J., et WILLIAMS Y. (2019). « Choice without inclusion? Comparing the intensity of racial segregation in charters and public schools at the local, state, and national levels ». Journal of Education Sciences, 9(205), p. 1-17.

VERGER A., FONTDEVILA C. et ZANCAJO A. (2016). The privatization of education: A political economy of global education reform. New York : Teachers College Press. 